Original article

\title{
Clinical laboratory characteristics of severe patients with coronavirus disease 2019 (COVID-19): A systematic review and meta-analysis
}

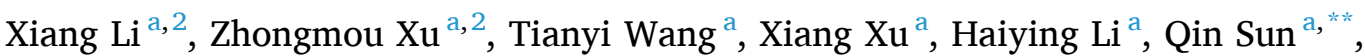 \\ Xinmin Zhou ${ }^{\mathrm{b}, *, 1}$, Gang Chen ${ }^{\mathrm{a}}$ \\ ${ }^{a}$ Department of Clinical Medicine, The First Affiliated Hospital of Soochow University, Suzhou, Jiangsu Province, China \\ ${ }^{\mathrm{b}}$ Department of Neurosurgery, The Affiliated Jiangyin Hospital, School of Medicine, Southeast University, Jiangyin, Jiangsu Province, China
}

\section{A R T I C L E I N F O}

\section{Keywords:}

SARS-CoV-2

Clinical characteristics

Disease severity

Meta-analysis

\begin{abstract}
A B S T R A C T
Objectives: To identify clinical characteristics of severe patients with COVID-19.

Methods: The WHO database of publications on COVID-19 and PubMed were searched from inception to March 20, 2020 and all valuable studies were analyzed using Stata 15.0.

Results: We selected forty-four studies with 13,497 patients. In the comparison of severe and non-severe groups, age over $50(\mathrm{OR}=4.090 ; 95 \% \mathrm{CI}=2.422-6.907, \mathrm{P}=0.000)$ and underlying disease $(\mathrm{OR}=3.992 ; 95 \% \mathrm{CI}=$ $2.631-6.507, \mathrm{P}=0.000)$ are risk factors. Female gender $(\mathrm{OR}=0.740 ; 95 \% \mathrm{CI}=0.622-0.881, \mathrm{P}=0.001)$ is a protective factor. Characteristics like dyspnea $(\mathrm{OR}=4.914 ; 95 \% \mathrm{CI}=3.069-7.867, \mathrm{P}=0.000$ ), lymphopenia $(\mathrm{OR}=5.528 ; 95 \% \mathrm{CI}=3.484-8.772, \mathrm{P}=0.000)$, thrombocytopenia $(\mathrm{OR}=3.623 ; 95 \% \mathrm{CI}=1.034-12.691, \mathrm{P}=$ $0.044)$, elevated C-reactive protein ( $\mathrm{OR}=5.217 ; 95 \% \mathrm{CI}=2.459-11.070, \mathrm{P}=0.000)$ and $\mathrm{D}$-dimer $(\mathrm{OR}=3.780$; $95 \% \mathrm{CI}=1.481-9.648, \mathrm{P}=0.005)$ were more frequently in severe cases. Diffuse lesions and consolidation (OR $=4.680 ; 95 \% \mathrm{CI}=3.183-6.881, \mathrm{P}=0.000$ ) in imaging was considered reliable.

Conclusions: Men older than 50 with underlying disease are susceptible to develop severe pneumonia while female gender is protective. The typical symptom of severe pneumonia was dyspnea, but high fever, headache and diarrhea were not significantly different among patients with varying degrees of severity. Lymphopenia, thrombocytopenia, elevated C-reactive protein and D-dimer occurred more frequently in severe patients and yet leukopenia is not a characteristic laboratory indicator. Diffuse lesions and consolidation are important imaging features to distinguish severe pneumonia.
\end{abstract}

\section{Introduction}

In December 2019, A group of unexplained pneumonia patients appeared in Wuhan, Hubei Province, China. And then a novel coronavirus was isolated from patients' airway epithelial cells which is the seventh member of the family of coronaviruses, named 2019-nCoV. ${ }^{1}$ The danger of 2019-nCoV is that it may resemble the Middle East respiratory syndrome coronavirus, which can cause severe respiratory diseases, including acute respiratory distress syndrome and respiratory failure. ${ }^{2}$ Even more dangerous is the strong person-to-person infectivity of the 2019-nCoV, which has spread rapidly worldwide. On March 11, 2020, The World Health Organization (WHO) has declared the novel coronavirus (COVID-19) outbreak a global pandemic, As of August 13, 2020 , there have been 89,526 confirmations In China resulting in 4704 deaths and 20,732,025confirmations in other countries, resulting in 748,068 deaths. ${ }^{3}$ These numbers are updated daily and are expected to continue growing.

A study from Washington, USA, found that $86 \%$ of critically ill patients admitted to ICU developed complications, $71 \%$ required mechanical ventilation, and as of March 17, 2020, the mortality rate was $67 \%$ and only $9.5 \%$ could be discharged. ${ }^{4}$ Similarly, a Chinese expert, academician Zhong, has shown that severe pneumonia is independently associated with admission to intensive care units, mechanical ventilation, or death (sub-distribution risk ratio, 9.80; The 95\% confidence

\footnotetext{
* Corresponding author.

** Corresponding author.

E-mail addresses: qsun@suda.edu.cn (Q. Sun), jeanne88@163.com (X. Zhou).

${ }^{1}$ Current Address: Department of Neurosurgery, The Affiliated Jiangyin Hospital, School of Medicine, Southeast University, Jiangyin, Jiangsu Province, China

2 These authors contributed equally to this work.
} 
Table 1

Characteristics of included studies.

\begin{tabular}{|c|c|c|c|c|c|c|}
\hline First author & Publication date & Composite endpoint & Region & Events patients & All patients & AXIS scores \\
\hline Jin-jin Zhang(12) & 2020-Feb & NA & China & NA & 140 & 13 \\
\hline Sijia Tian(13) & 2020-Feb & Death & China & 3 & 241 & 14 \\
\hline Lei Chen(7) & 2020-Feb & Death & China & 2 & 29 & 15 \\
\hline Wenjie Yang(14) & 2020-Feb & IMV & China & 2 & 149 & 16 \\
\hline Chaolin Huang(2) & 2020-Feb & ICU & China & 13 & 41 & 16 \\
\hline Wei Liu(15) & 2020-Feb & Death & China & 2 & 78 & 14 \\
\hline Kunhua Li(16) & 2020-Feb & NA & China & NA & 83 & 16 \\
\hline Jian Wu(17) & 2020-Feb & Death & China & 0 & 80 & 14 \\
\hline Yihui Huan(18) & 2020-Feb & IMV & China & 3 & 34 & 13 \\
\hline Weijie Guan(5) & $2020-\mathrm{Feb}$ & ICU & China & 55 & 1099 & 16 \\
\hline Kin On Kwok(19) & 2020-Feb & ICU & China & 3 & 14 & 13 \\
\hline Kui Liu(20) & 2020-Feb & Death & China & 16 & 137 & 14 \\
\hline Ning Tang(21) & 2020-Feb & Death & China & 21 & 183 & 16 \\
\hline De Chang(22) & 2020-Feb & ICU & China & 0 & 13 & 13 \\
\hline Dawei Wang(23) & 2020-Feb & ICU & China & 36 & 138 & 16 \\
\hline Xiaowei Xu(24) & 2020-Feb & ICU & China & 1 & 62 & 16 \\
\hline Nanshan Chen(25) & 2020-Feb & Death & China & 11 & 99 & 16 \\
\hline Yu-Huan Xu(26) & 2020-Feb & ICU & China & 3 & 50 & 15 \\
\hline SB Stoecklin(43) & 2020-Feb & ICU & France & 1 & 3 & 15 \\
\hline Albarello Fabrizio(44) & 2020-Feb & Death & Italy & 21 & 888 & 13 \\
\hline Daniel B. Jernigan(45) & 2020-Feb & Death & USA & 0 & 53 & 15 \\
\hline KSOID(47) & 2020-Mar & Death & Korea & 22 & 4212 & 15 \\
\hline ZhongliangWang(27) & 2020-Mar & Death & China & 5 & 69 & 14 \\
\hline Barnaby E Young(50) & 2020-Mar & IMV & Singapore & 2 & 36 & 16 \\
\hline Pingzheng $\mathrm{Mo}(28)$ & 2020-Mar & NA & China & NA & 155 & 16 \\
\hline Chuan Qin(29) & 2020-Mar & NA & China & NA & 452 & 14 \\
\hline Yichun Cheng(30) & 2020-Mar & Death & China & 113 & 701 & 16 \\
\hline Yudong Peng(31) & 2020-Mar & Death & China & 17 & 112 & 16 \\
\hline CDCT(46) & 2020-Mar & ICU & USA & 121 & 2449 & 13 \\
\hline Fei Zhou(32) & 2020-Mar & Death & China & 54 & 191 & 16 \\
\hline Yu Shi(33) & 2020-Mar & Death & China & 0 & 487 & 13 \\
\hline Mingli Yuan(34) & 2020-Mar & Death & China & 10 & 27 & 14 \\
\hline Tianmin $\mathrm{Xu}(35)$ & 2020-Mar & ICU & China & 0 & 51 & 16 \\
\hline Jun Chen(36) & 2020-Mar & ICU & China & 22 & 249 & 14 \\
\hline Bingwen Eugene FAN(49) & 2020-Mar & ICU & Singapore & 9 & 67 & 14 \\
\hline Qiurong Ruan(37) & 2020-Mar & NA & China & NA & 150 & 15 \\
\hline BE Young(50) & 2020-Mar & ICU & Singapore & 2 & 18 & 16 \\
\hline Wei Zhao(38) & 2020-Mar & NA & China & NA & 101 & 16 \\
\hline Yingxia Liu(39) & 2020-Mar & IMV & China & 3 & 12 & 13 \\
\hline Liu Min(40) & 2020-Mar & NA & China & NA & 30 & 15 \\
\hline Zhenwei Wang(41) & 2020-Mar & IMV & China & 1 & 4 & 13 \\
\hline Chaomin Wu(42) & 2020-Mar & ICU & China & 53 & 201 & 16 \\
\hline CNIRSTOA(51) & 2020-Mar & Death & Australia & 2 & 71 & 13 \\
\hline Gianfranco Spiteri(52) & 2020-Mar & ICU & Europe & 3 & 38 & 13 \\
\hline
\end{tabular}

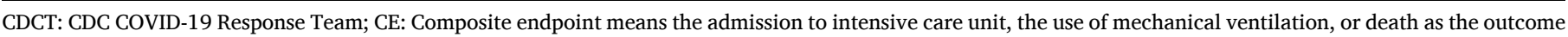

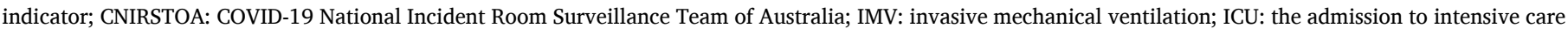
unit; KSOID: Korean Society of Infectious Diseases NA: No data available.

interval, 4.06-23.67), provides a composite endpoint for poor prognosis. ${ }^{5}$ In the face of this epidemic, the rational allocation of medical resources is particularly important. Classification of patients' conditions and active rescue of severe patients can minimize the case fatality rate and poor prognosis rate, what's worse, there is insufficient data about severe pneumonia with OVID-19. Here, we searched the observational studies that mentioned the composite endpoint (admission to intensive care units, the use of mechanical ventilation, or death) of poor prognosis among all confirmed patient, analyzed the overall rate of poor prognosis, and then revealed the clinical characteristics and indicators directly related to severe pneumonia so as to provide help for clinical treatment and control of COVID-19.

\section{Methods}

\subsection{Data sources and searches}

WHO is gathering the latest scientific findings and knowledge on COVID-19, and compiling it in a database and they update the database daily from searches of bibliographic databases. We downloaded the latest COVID-19 study summary table on the WTO platform on March 20,2020 , and then reviewed the corresponding studies and extracted the data for analysis according to the summary table. Since the earliest COVID-19 was reported in Wuhan, China, in December 2019, the retrieval time was limited after this time. We searched published articles without any language limit. In order to avoid missing any valuable articles, we also manually searched on PubMed using a combination of the following terms: novel Coronavirus Pneumonia; 2019-nCoV; COVID-19; SARS-CoV-2; clinical characteristics; clinical features and we also carefully scrutinized references of these studies to identify other useful studies.

\subsection{Study selection}

Due to the particularity of the COVID-19, the articles published in the database have a certain timeliness. In order to calculate the overall poor prognosis rate and avoid missing useful information, articles mentioned the composite endpoint are included in the primary meta-analysis and valuable graded clinical data was further combined and analyzed. The included study types include retrospective case-control study, case report, case series, etc. Some studies which did not explicitly mention the endpoints, but compared severe and non-severe patients were also included for further study. 
(A)

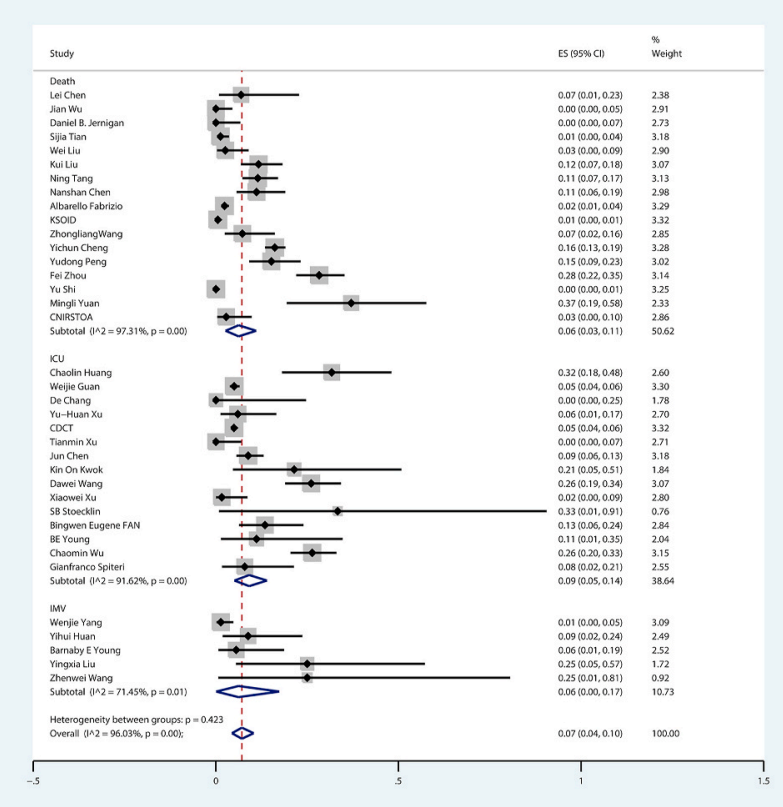

(C)

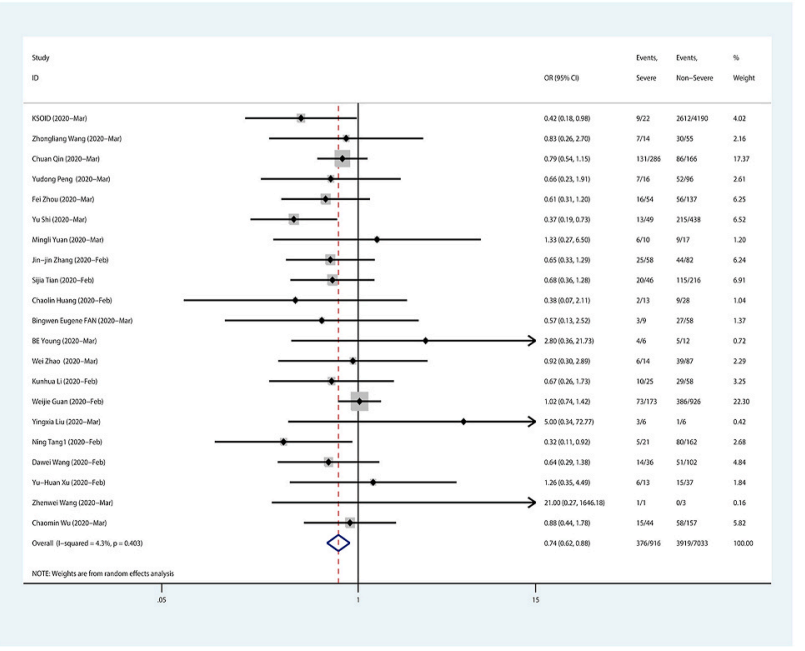

(B)

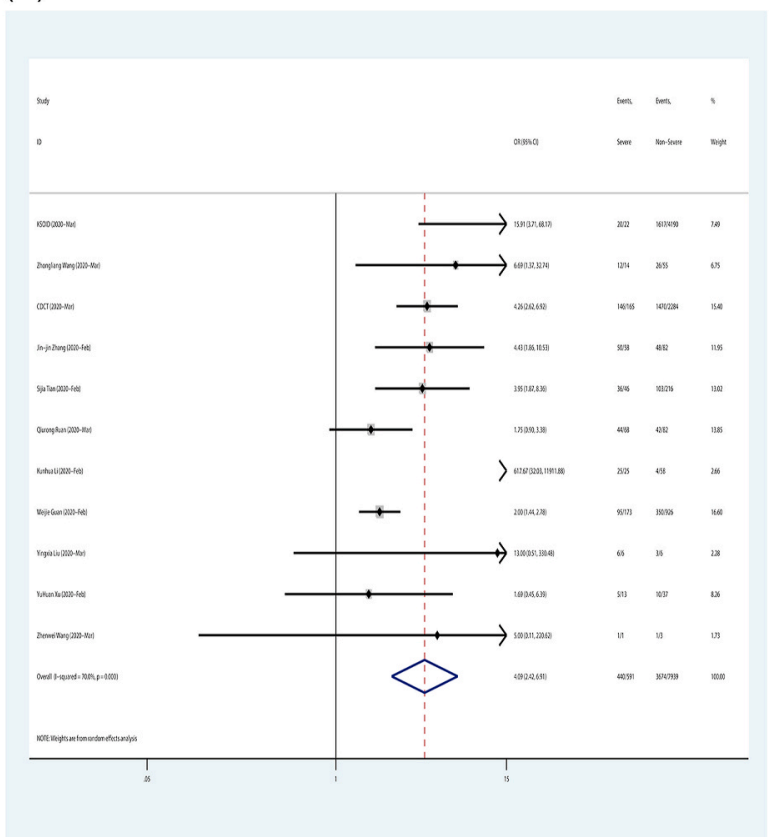

(D)

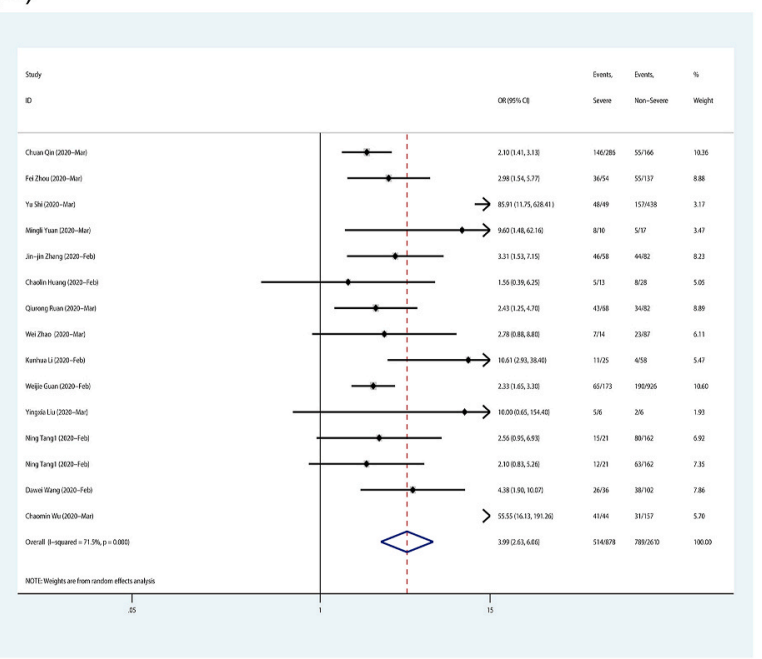

Fig. 1. Forest plot for composite endpoint incidence in all patients and demographic information of severe patients compared with non-severe patients. (A) composite endpoint incidence; (B) The age over 50 years old; (C) The proportion of female; (D) With any underlying diseases.

\subsection{Classification of the patients}

According to the current international guidelines and the Chinese National Health Committee covid-19 clinical practice, ${ }^{6,7}$ we divided the confirmed patients into severe and non-severe groups. The patients of the non-severe group were defined as having fever, respiratory tract symptoms, etc., and even imaging showed pneumonia. The severe group was defined as having with any of the following conditions ${ }^{1}$ : respiratory distress, $\mathrm{RR} \geq 30$ times $/ \mathrm{min}^{2}$; In the resting state, refers to the oxygen saturation $\leq 93 \%$; Arterial partial oxygen pressure $\left(\mathrm{PaO}_{2}\right)$ /oxygen absorption concentration $\left(\mathrm{FiO}_{2}\right) \leq 300 \mathrm{mmHg}(1 \mathrm{mmHg}=0.133 \mathrm{kpa})^{4}$; respiratory failure and the need for mechanical ventilation; ${ }^{5}$ Shock or non-survivor ${ }^{6}$ Combined with other organ failure requires ICU care.

\subsection{Data extraction and quality assessment}

The following all available information were extracted, including the first author, publication year, region, the number of all patients, the number of patients with composite endpoint, all valuable clinic data. We assessed the quality of all included studies using the Appraisal tool for Cross-Sectional Studies (AXIS). ${ }^{8}$ All data were separately extracted by 2 reviewers and integrated clearly.

\subsection{Data synthesis and analysis}

Stata version 15.0 was used for all meta-analysis to evaluate pooled effect values, such as odds ratios. Heterogeneity among studies was calculated and assessed using the Chi-square test and $\mathrm{I}^{2}$ statistic. Different ranges of $\mathrm{I}^{2}$ reflect the effects of heterogeneity on the predictive value of meta-analyses. When $\mathrm{I}^{2}<25 \%$ means low heterogeneity; $\mathrm{I}^{2}$ $=25 \%-50 \%$ means moderate heterogeneity; $\mathrm{I}^{2}>50 \%$ means high heterogeneity. ${ }^{9}$ When $\mathrm{I}^{2}>50 \%$, we chose the random-effects model to analyze. In order to assess the influence of every individual study to the pooled results, one study was excluded in sequence to the sensitivity analysis and if there was statistical heterogeneity, sensitivity analysis would determine the source of heterogeneity. ${ }^{10}$ We assessed publication bias using funnel plots, by Begg's adjusted rank correlation test. ${ }^{11}$ Statistical tests were two-sided and used a significance level of $\mathrm{p}<0.05$. 
(A)

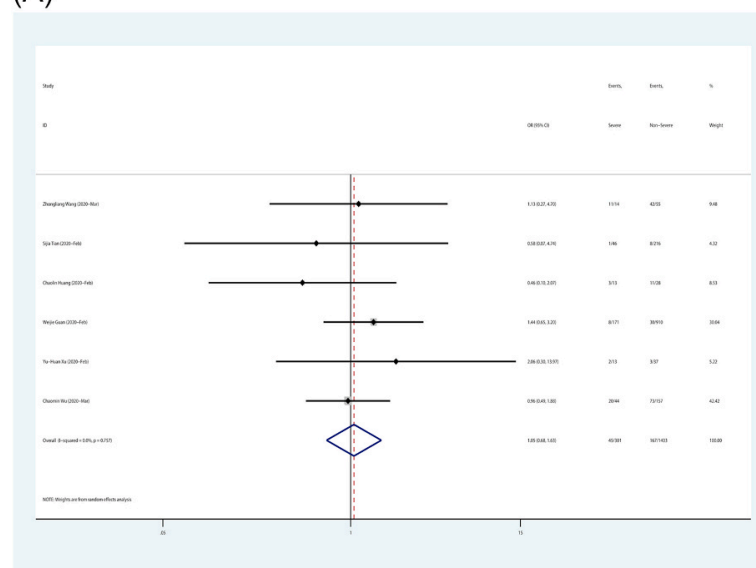

(C)

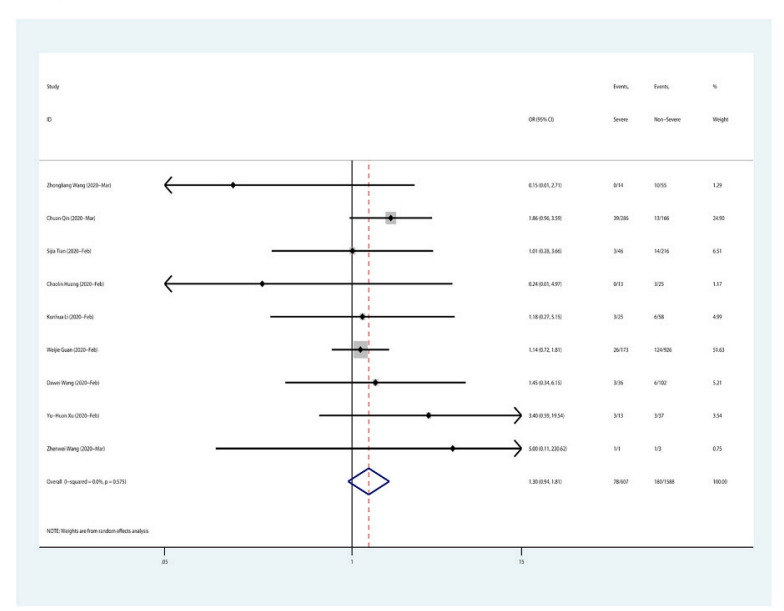

(B)

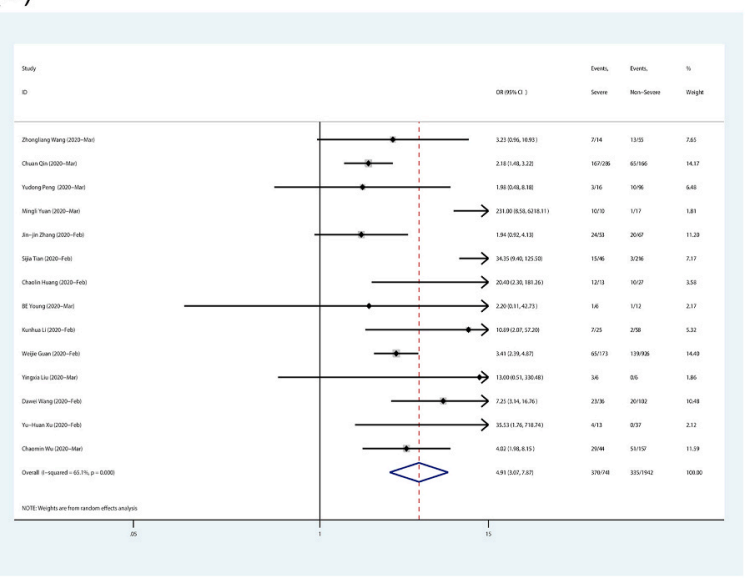

(D)

Fig. 2. Forest plot for clinical characteristics of severe patients compared with non-severe patients. (A) High fever: body temperature is over $39^{\circ}$ Celsius; (B) Dyspnea; (C)Headache (D)diarrhea.

\section{Results}

\subsection{Study selection}

We searched 2207 studies from the online database of WHO and 1 through manual search. After removing duplicate, 2133 related articles were included for further screening. After reviewing the titles and abstracts 2040 articles were excluded and the left 93 articles were chosen for full-text assessment, 49 studies were further excluded for the following reasons: no enough grading data to estimate the severe and non-severe groups or no mention of composite endpoint information, the population data reusing. Finally, 44 studies were selected in metaanalysis and the process was showed in Supplementary Fig. 1.

\subsection{Study characteristics}

These studies were all published in February and March 2020. Most of the study population came from China since the early period of the outbreak occurred in China. 34 studies included in our meta-analysis were in China. ${ }^{2,5,7,12-42} 1$ study was based in France ${ }^{43}$ and 1 in Italy, ${ }^{44} 2$ in USA, ${ }^{45,46} 1$ study in Korea, ${ }^{47} 3$ in Singapore, ${ }^{48-50} 1$ in Australia ${ }^{51}$ and 1 study were conducted in European. ${ }^{52}$ A total of 13,497 patients were enrolled in this study, and during the follow-up period, 632 reached the composite endpoint, which means the admission to an intensive care unit, the use of mechanical ventilation, or death. Detailed information was presented in Table 1.

\subsection{Overall composite endpoint incidence of COVID-19 in all confirmed patients}

According to previous studies, ${ }^{4,5}$ the composite endpoint can indicate a poor prognosis. We extracted and analyzed the endpoints of all enrolled patients as shown in Fig. 1 (A). The combined mortality was 0.06 (95\% CI $0.03-0.11, \mathrm{P}=0.00$ ) and the combined rate of the admission to the intensive care unit was 0.09 (95\% CI 0.05-0.14, P = 0.00 ), the combined rate of mechanical ventilation treatment was 0.06 (95\% CI 0.00-0.0.17, $\mathrm{P}=0.03$ ). The overall composite endpoint rate of COVID-19 was 0.07 ( $95 \%$ CI $0.04-0.10, \mathrm{P}=0.00$ ), which means a poor prognosis in about $7 \%$ of diagnosed patients.

The analysis of demographic information and clinical characteristics of severe patients compared with non-severe patients.

We extracted and analyzed the demographic information and clinical characteristics of severe and non-severe patients. The combined odds ratio (OR) for patients over 50 years old of severe patients compared with non-severe patients was 4.090 (95\% CI 2.422-6.907, $\mathrm{P}=0.000$ ) (Fig. 1 (B)). This result indicates that patients over 50 are more susceptible to become severe. The combined OR of female gender of severe patients compared with non-severe patients was 0.740 (95\% CI 0.622-0.881, $\mathrm{P}=0.001$ ) (Fig. $1(\mathrm{C})$ ), which revealed femaleness may be a protective factor. The pooled $\mathrm{OR}$ value of the underlying disease is 3.992 (95\% CI 2.631-6.057, $\mathrm{P}=0.000$ ) (Fig. 1 (D)), this value suggests patients with any underlying disease were more vulnerable to severe pneumonia.

In terms of clinical characteristics, we analyzed high fever, dyspnea, 
(A)

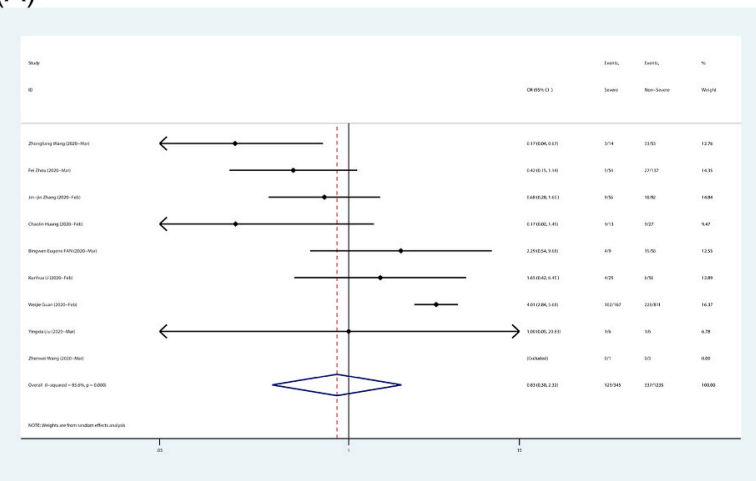

(C)

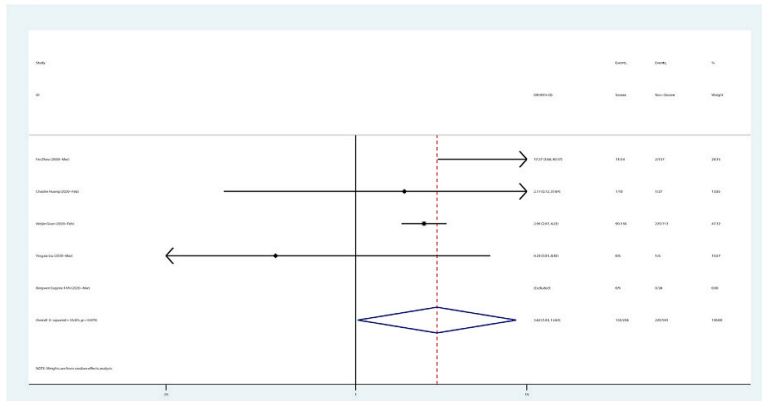

$(\mathrm{E})$

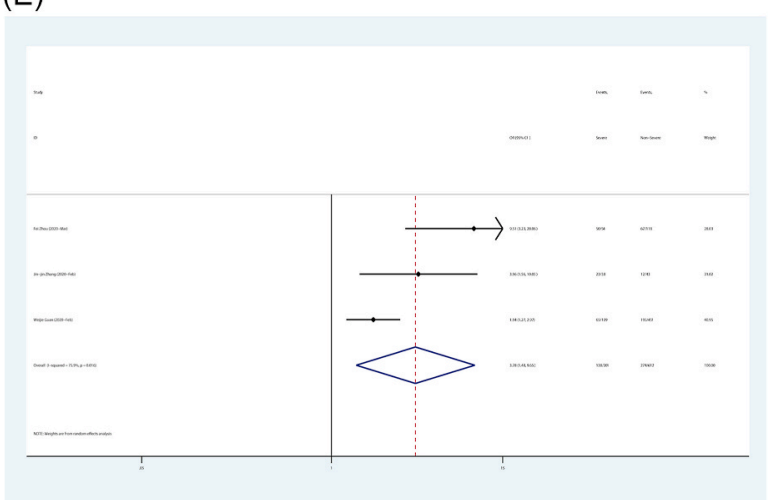

(B)

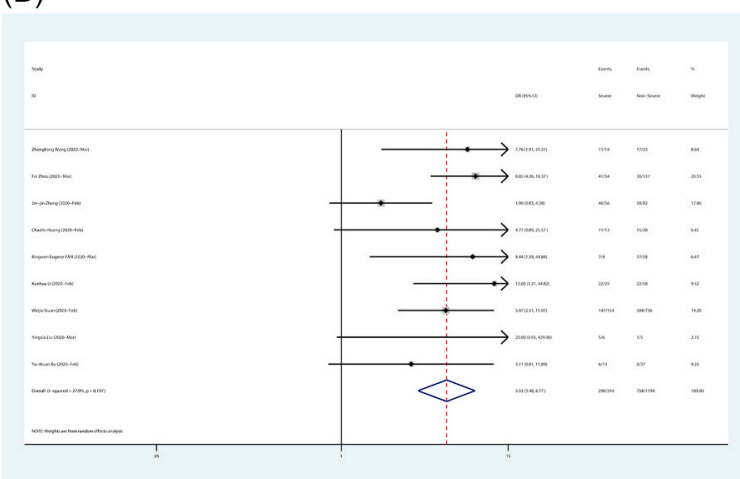

(D)

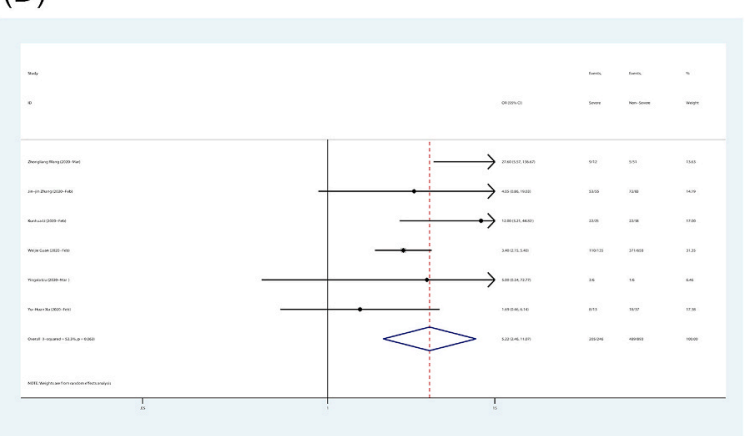

(F)

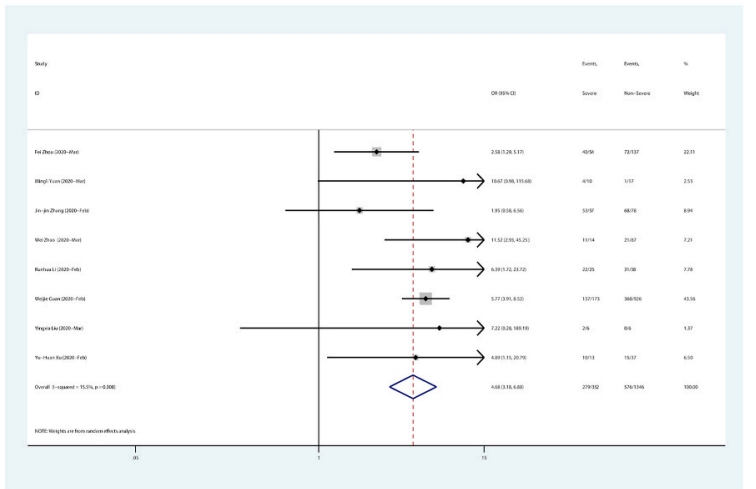

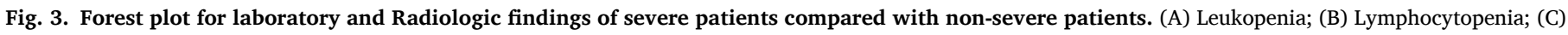
Thrombocytopenia; (D) Elevated c-reactive proteins (E) Elevated D-dimers; (F) Radiologic findings: diffuse lesions and consolidation.

headache and diarrhea as typical symptoms of the respiratory, nervous system and digestive system respectively. We analyzed the number of patients admitted to the hospital with a body temperature higher than $39^{\circ}$ Celsius in two groups, the combined OR was 1.053 (95\% CI $0.680-1.631, \mathrm{P}=0.815$ ) (Fig. 2 (A)). Dyspnea was the main assessment symptom, and we analyzed the number of patients with dyspnea in two groups, the pooled OR was $4.914(95 \%$ CI $3.069-7.867, \mathrm{P}=0.000)$ (Fig. 2 (B)), and the combined OR of headache and diarrhea was 1.305 (95\% CI 0.939-1.813, P = 0.113) and 1.205 (95\% CI 0.892-1.628, P = 0.224 ) respectively (Fig. 2 (C),(D)). These results showed that dyspnea is the most valuable symptom for prognostic classification, however, high fever, headache and diarrhea are not typical.

\subsection{The analysis of laboratory and radiologic findings of severe patients compared with non-severe patients}

Fig. 3 shows the laboratory and radiologic findings analysis, we still divided the patients into severe and non-severe groups. Leukopenia, lymphopenia and thrombocytopenia have been observed in some patients, ${ }^{5}$ so we extracted the number of patients with this laboratory test in both groups and performed a combined analysis, the combined OR of leukopenia in severe patients compared with non-severe patients was 0.830 ( $95 \%$ CI $0.298-2.316, \mathrm{P}=0.722$ ) (Fig. $3(\mathrm{~A})$ ) and the combined OR of lymphopenia and thrombocytopenia was 5.528 (95\% CI 3.484-8.772, $\mathrm{P}=0.000)$ and 3.623 (95\% CI 1.034-12.691, $\mathrm{P}=0.044)$ (Fig. 3 (B),(C)). And we further analyzed the common inflammatory markers C-reactive protein and D-dimer. The pooled OR of elevated C-reactive protein in severe and non-severe groups was 5.217 (95\% CI 2.459-11.070, $\mathrm{P}=0.000$ ) (Fig. 3 (D)) and the pooled OR of elevated D-dimer was 3.780 (95\% CI 1.481-9.648, P = 0.005) (Fig. 3 (E)). These results suggest that lymphocytopenia, thrombocytopenia and the elevated C-reactive protein and D-dimer were closely associated with severe pneumonia, however, leukopenia was not representative in severe patients. As for the radiologic findings, we mainly analyzed diffuse lesions and consolidation. The pooled OR of severe patients compared with non-severe patients was 4.680 (95\% CI 3.183-6.881, $\mathrm{P}=0.000$ ) (Fig. 3 (F)). These results obviously showed radiologic findings were the most direct diagnostic method for severe pneumonia. 


\subsection{Sensitivity analysis and publication bias analysis}

In Supplementary Fig. 2, sensitivity analyses were performed to evaluate the influence of individual study on the overall results through omitting study one by one. We performed sensitivity analyses for each pooled result, some of which are shown in Supplementary Fig. 2 (A), The results showed there are no meaningful change after omitting any study. As shown in Supplementary Fig. 2 (B), the funnel plot does not show obvious asymmetry, the $\mathrm{p}$ value for Begg's test was 0.244 which also suggested no significant publication bias.

\section{Discussion}

Our study indicated that the overall poor prognosis rate of COVID 19 was about $7 \%$, which is consistent with other relevant studies. The mortality rate was higher than reported, which may be explained by Berkson's bias that severe patients tend to get priority hospitalization. The rate of treatment with mechanical ventilation was $6 \%$, which may be lower than that of some other studies, possibly due to incomplete data on patients using mechanically ventilated included in our analysis. It is also possible that some patients who need mechanical ventilation do not receive it due to the shortage of ventilators. We grouped patients with severe and non-severe pneumonia to reveal differences in clinical characteristics, laboratory examination and radiologic findings. This analysis showed that men over the age of 50 with underlying disease were more likely to progress to severe pneumonia. Older age and underlying disease are easily explained as risk factors. Female gender may be a protective factor, which may be related to the gender specificity of women, who have fewer bad habits than men, especially smoking. ${ }^{53}$ There are some studies suggested COVID-19 has infected more males than females. ${ }^{54}$ Females could develop enhanced innate and adaptive immune responses than males which make them less susceptible to many infections of viral, bacterial, parasitic, and fungal origin. ${ }^{55,56}$ Dyspnea may be the primary clinical symptom to determine the severity of COVID-19, while the associated symptoms of high fever, headache and diarrhea were not significantly different between severe and the non-severe group.

As for laboratory tests, the incidence of lymphocytopenia and thrombocytopenia is significantly increased in patients with severe pneumonia, and the levels of C-reactive protein and D-dimer were also elevated. However, Leukopenia may not be a characteristic laboratory finding of severe pneumonia. The underlying pathogenesis of COVID-19 is still unknown. There are some studies showed inflammatory storms and damage to cellular immunity may play an important role in severe pneumonia. ${ }^{57}$ And older patients are more likely to develop severe pneumonia, perhaps due to their weakened immune systems. ${ }^{58}$ Imaging as the most direct method to identify the degree of disease is accurate and effective, diffuse lesions and consolidation are features of severe pneumonia. ${ }^{59}$

This analysis has several limitations. First of all, COVID-19 is spreading rapidly, and epidemiological data are being updated rapidly, due to the lag of scientific research papers and clinical studies, some data only represent the characteristics of the early epidemic. Some valuable case information from Europe, America and even Africa were not available, resulting in regional limitation. A larger study of patients with COVID-19 pneumonia from other countries would help to further define the clinical characteristics and risk factors of the disease. Second, all data included in this study were from the official information of the hospital, some patients cannot be hospitalized for treatment because of the shortage of medical resources. The loss of this part of data may lead to the underrepresentation of the collected cases. Third, due to the particularity of covid-19, the clinical data included in the articles were quite different, resulting in great heterogeneity. Therefore, the random effect model was adopted in this study, which poses a challenge to the partial results of this study and requires further study in larger and higher-quality studies.

\section{Conclusion}

The overall Composite endpoint incidence of COVID 19 was about $7 \%$, the mortality rate was $6 \%$, ICU admission rate was $9 \%$, and mechanical ventilation rate was $6 \%$, patients older than 50 with underlying disease were susceptible to develop severe pneumonia and female gender was a protective factor. The typical symptom of severe pneumonia was dyspnea while high fever, headache and diarrhea were not significantly different between severe and the non-severe groups. Lymphocytopenia, thrombocytopenia, elevated c-reactive proteins and Ddimers are more common in critically ill patients, however, leukopenia is not a characteristic laboratory indicator. Diffuse lesions and consolidation are important imaging features to distinguish severe pneumonia.

\section{Declaration of competing interest}

The authors declare that the research was conducted in the absence of any commercial or financial relationships that could be construed as a potential conflict of interest.

\section{Acknowledgment}

Thank you to all those who have contributed to fighting the COVID19 outbreak. This research did not receive any specific grant from funding agencies in the public, commercial, or not-for-profit sectors.

\section{Appendix A. Supplementary data}

Supplementary data to this article can be found online at https://doi. org/10.1016/j.cegh.2020.08.012.

\section{References}

1 Zhu N, Zhang D, Wang W, et al. A novel coronavirus from patients with pneumonia in China, 2019. N Engl J Med. 2020;382(8):727-733.

2 Huang C, Wang Y, Li X, et al. Clinical features of patients infected with 2019 novel coronavirus in Wuhan, China. Lancet. 2020;395(10223):497-506.

3 Cucinotta D, Vanelli M. WHO declares COVID-19 a pandemic. Acta Biomed: Atenei Parmensis. 2020;91(1):157-160.

4 Arentz M, Yim E, Klaff L, et al. Characteristics and outcomes of 21 critically ill patients with COVID-19 in Washington state. Jama. 2020;326(16):1612-1614.

5 Guan WJ, Ni ZY, Hu Y, et al. Clinical characteristics of coronavirus disease 2019 in China. N Engl J Med. 2020;382(18):1708-1720.

6 Metlay JP, Waterer GW, Long AC, et al. Diagnosis and treatment of adults with community-acquired pneumonia. An official clinical practice guideline of the American thoracic society and infectious diseases society of America. Am J Respir Crit Care Med. 2019;200(7):e45-e67.

7 Chen L, Liu HG, Liu W, et al. Analysis of clinical features of 29 patients with 2019 novel coronavirus pneumonia. Zhonghua jie he he hu xi za zhi=Zhonghua jiehe he huxi zazhi $=$ Chin J Tuberc Respir Dis. 2020;43(3):203-208.

8 Downes MJ, Brennan ML, Williams HC, Dean RS. Development of a critical appraisal tool to assess the quality of cross-sectional studies (AXIS). BMJ open. 2016;6(12), e011458.

9 Melsen WG, Bootsma MC, Rovers MM, Bonten MJ. The effects of clinical and statistical heterogeneity on the predictive values of results from meta-analyses. Clin Microbiol Infect : Off Publ Eur Soc Clin Microbiol Infect Dis. 2014;20(2):123-129.

10 Copas J, Shi JQ. Meta-analysis, funnel plots and sensitivity analysis. Biostatistics. 2000;1(3):247-262.

11 Choi SW, Lam DM. Funnels for publication bias-have we lost the plot? Anaesthesia. 2016;71(3):338-341.

12 Zhang JJ, Dong X, Cao YY, et al. Clinical characteristics of 140 patients infected with SARS-CoV-2 in Wuhan, China. Allergy. 2020;75(7):1730-1741.

13 Tian S, Hu N, Lou J, et al. Characteristics of COVID-19 infection in Beijing. J Infect. 2020;80(4):401-406.

14 Yang W, Cao Q, Qin L, et al. Clinical characteristics and imaging manifestations of the 2019 novel coronavirus disease (COVID-19):A multi-center study in Wenzhou city, Zhejiang, China. J Infect. 2020;80(4):388-393.

15 Liu W, Tao ZW, Lei W, et al. Analysis of factors associated with disease outcomes in hospitalized patients with 2019 novel coronavirus disease. Chinese Med J. 2020;133 (9):1032-1038.

16 Li K, Wu J, Wu F, et al. The clinical and chest CT features associated with severe and critical COVID-19 pneumonia. Invest Radiol. 2020;55(6):327-331.

17 Wu J, Liu J, Zhao X, et al. Clinical Characteristics of Imported Cases of COVID-19 in Jiangsu Province: A Multicenter Descriptive Study. In: Clinical infectious diseases : an official publication of the Infectious Diseases Society of America. 71. 2020:706-712. 
18 Huang Y, Tu M, Wang S, et al. Clinical characteristics of laboratory confirmed positive cases of SARS-CoV-2 infection in Wuhan, China: a retrospective single center analysis. Trav Med Infect Dis. 2020:101606.

19 Kwok KO, Wong V, Wei VWI, Wong SYS, Tang JW. Novel coronavirus (2019-nCoV) cases in Hong Kong and implications for further spread. J Infect. 2020;80(6): $671-693$.

20 Liu K, Fang YY, Deng Y, et al. Clinical characteristics of novel coronavirus cases in tertiary hospitals in Hubei Province. Chinese Med J. 2020;133(9):1025-1031.

21 Tang N, Li D, Wang X, Sun Z. Abnormal coagulation parameters are associated with poor prognosis in patients with novel coronavirus pneumonia. $J$ Thromb Haemostasis : JTH. 2020;18(4):844-847.

22 Chang Lin M, Wei L, Xie L, Zhu G, Dela Cruz CS, et al. Epidemiologic and clinical characteristics of novel coronavirus infections involving 13 patients outside Wuhan, China. Jama. 2020;323(11):1092-1093.

23 Wang D, Hu B, Hu C, et al. Clinical characteristics of 138 hospitalized patients with 2019 novel coronavirus-infected pneumonia in Wuhan, China. Jama. 2020;323(11): $1061-1069$.

24 Xu XW, Wu XX, Jiang XG, et al. Clinical findings in a group of patients infected with the 2019 novel coronavirus (SARS-Cov-2) outside of Wuhan, China: retrospective case series. Bmj. 2020;368:m606.

25 Chen N, Zhou M, Dong X, et al. Epidemiological and clinical characteristics of 99 cases of 2019 novel coronavirus pneumonia in Wuhan, China: a descriptive study. Lancet. 2020;395(10223):507-513.

$26 \mathrm{Xu} \mathrm{YH,} \mathrm{Dong} \mathrm{JH,} \mathrm{An} \mathrm{WM,} \mathrm{et} \mathrm{al.} \mathrm{Clinical} \mathrm{and} \mathrm{computed} \mathrm{tomographic} \mathrm{imaging} \mathrm{features}$ of novel coronavirus pneumonia caused by SARS-CoV-2. J Infect. 2020;80(4): 394-400.

27 Wang Z, Yang B, Li Q, Wen L, Zhang R. Clinical Features of 69 Cases with Coronavirus Disease 2019 in Wuhan, China. Clinical infectious diseases : an official publication of the Infectious Diseases Society of America; 2020.

28 Mo P, Xing Y, Xiao Y, et al. Clinical Characteristics of Refractory COVID-19 Pneumonia in Wuhan, China. Clinical infectious diseases : an official publication of the Infectious Diseases Society of America; 2020.

29 Qin C, Zhou L, Hu Z, et al. Dysregulation of Immune Response in Patients with COVID-19 in Wuhan, China. Clinical infectious diseases : an official publication of the Infectious Diseases Society of America; 2020.

30 Cheng Y, Luo R, Wang K, et al. Kidney disease is associated with in-hospital death of patients with COVID-19. Kidney Int. 2020;97(5):829-838.

31 Peng YD, Meng K, Guan HQ, et al. [Clinical characteristics and outcomes of 112 cardiovascular disease patients infected by $2019-\mathrm{nCoV}]$. Zhonghua Xinxueguanbing Zazhi. 2020;48:E004, 0.

32 Zhou F, Yu T, Du R, et al. Clinical course and risk factors for mortality of adult inpatients with COVID-19 in Wuhan, China: a retrospective cohort study. Lancet. 2020;395(10229):1054-1062.

33 Shi Y, Yu X, Zhao H, Wang H, Zhao R, Sheng J. Host susceptibility to severe COVID19 and establishment of a host risk score: findings of 487 cases outside Wuhan. Crit Care. 2020;24(1):108.

34 Yuan M, Yin W, Tao Z, Tan W, Hu Y. Association of radiologic findings with mortality of patients infected with 2019 novel coronavirus in Wuhan, China. PloS One. 2020;15 (3), e0230548.

$35 \mathrm{Xu}$ T, Chen C, Zhu Z, et al. Clinical features and dynamics of viral load in imported and non-imported patients with COVID-19. Int J Infect Dis: IJID: Off Publ Int Soc Infect Dis. 2020;94:68-71.

36 Chen J, Qi T, Liu L, et al. Clinical progression of patients with COVID-19 in Shanghai, China. J Infect. 2020;80(5):e1-e6.

37 Ruan Q, Yang K, Wang W, Jiang L, Song J. Clinical predictors of mortality due to COVID-19 based on an analysis of data of 150 patients from Wuhan, China. Intensive Care Med. 2020;46(6):1294-1297.

38 Zhao W, Zhong Z, Xie X, Yu Q, Liu J. Relation between chest CT findings and clinical conditions of coronavirus disease (COVID-19) pneumonia: a multicenter study. AJR Am J Roentgenol. 2020:1-6.
39 Liu Y, Yang Y, Zhang C, et al. Clinical and biochemical indexes from 2019-nCoV infected patients linked to viral loads and lung injury. Sci China Life Sci. 2020;63(3): 364-374.

40 Liu M, He P, Liu HG, et al. Clinical characteristics of 30 medical workers infected with new coronavirus pneumonia. Zhonghua jie he he hu xi za zhi=Zhonghua jiehe he huxi zazhi = Chin J Tuberc Respir Dis. 2020;43(3):209-214.

41 Wang Z, Chen X, Lu Y, Chen F, Zhang W. Clinical characteristics and therapeutic procedure for four cases with 2019 novel coronavirus pneumonia receiving combined Chinese and Western medicine treatment. Biosci. Trends. 2020;14(1): 64-68.

42 Wu C, Chen X, Cai Y, et al. Risk factors associated with acute respiratory distress syndrome and death in patients with coronavirus disease 2019 pneumonia in Wuhan, China. JAMA Intern Med. 2020;180(7):934-943.

43 Bernard Stoecklin S, Rolland P, Silue Y, et al. First cases of coronavirus disease 2019 (COVID-19) in France: surveillance, investigations and control measures. Euro Surveill : Bull Eur sur les Maladies Transmissibles = Eur Commun Dis Bull. January 2020; 25(6), 2020.

44 Porcheddu R, Serra C, Kelvin D, Kelvin N, Rubino S. Similarity in case fatality rates (CFR) of COVID-19/SARS-COV-2 in Italy and China. J Infect Dev Ctries. 2020;14(2): 125-128.

45 Jernigan DB, Team CC-R. Update: public Health response to the coronavirus disease 2019 outbreak - United States, February 24, 2020. MMWR Morb Mortal Wkly Rep. 2020;69(8):216-219.

46 CDC COVID-19 Response Team. Severe Outcomes Among Patients with Coronavirus Disease 2019 (COVID-19) — United States. Morb Mortal Wkly Rep. 18-March 2020. pdf.

47 Korean Society of Infectious D, Korean Society of Pediatric Infectious D, Korean Society of E, Korean Society for Antimicrobial T, Korean Society for Healthcareassociated Infection C, Prevention, et al. Report on the epidemiological features of coronavirus disease 2019 (COVID-19) outbreak in the Republic of Korea from January 19 to March 2, 2020. J Kor Med Sci. 2020;35(10):e112.

48 Pung R, Chiew CJ, Young BE, et al. Investigation of three clusters of COVID-19 in Singapore: implications for surveillance and response measures. Lancet. 2020;395 (10229):1039-1046.

49 Fan BE, Chong VCL, Chan SSW, et al. Hematologic parameters in patients with COVID-19 infection. Am J Hematol. 2020;95(6):e131-e134.

50 Young BE, Ong SWX, Kalimuddin S, et al. Epidemiologic features and clinical course of patients infected with SARS-CoV-2 in Singapore. Jama. 2020;323(15):1488-1494.

51 Team C-NIRS. COVID-19, Australia: Epidemiology Report 6 (Reporting Week Ending 19: 00 AEDT 7 March 2020). 44. Communicable diseases intelligence; 2020.

52 Spiteri G, Fielding J, Diercke M, et al. First cases of coronavirus disease 2019 (COVID-19) in the WHO European region, 24 January to 21 February 2020. Euro Surveill : Bull Eur sur les Maladies Transmissibles = Eur Commun Dis Bull. 2020;25(9).

53 Manavi KR, Alston-Mills BP, Thompson MP. History of tobacco, vitamin D and women. Int J Vitam Nutr Res. 2020:1-6.

54 Novel Coronavirus Pneumonia Emergency Response Epidemiology T. The epidemiological characteristics of an outbreak of 2019 novel coronavirus diseases (COVID-19) in China. Zhonghua liu xing bing xue za zhi = Zhonghua liuxingbingxue zazhi. 2020;41(2):145-151.

55 Jaillon S, Berthenet K, Garlanda C. Sexual dimorphism in innate immunity. Clin Rev Allergy Immunol. 2019;56(3):308-321.

56 Channappanavar R, Fett C, Mack M, Ten Eyck PP, Meyerholz DK, Perlman S. Sexbased differences in susceptibility to severe acute respiratory syndrome coronavirus infection. J Immunol. 2017;198(10):4046-4053.

57 Channappanavar R, Perlman S. Pathogenic human coronavirus infections: causes and consequences of cytokine storm and immunopathology. Semin Immunopathol. 2017; 39(5):529-539.

58 Goronzy JJ, Fang F, Cavanagh MM, Qi Q, Weyand CM. Naive T cell maintenance and function in human aging. J Immunol. 2015;194(9):4073-4080.

59 Wu J, Wu X, Zeng W, et al. Chest CT findings in patients with corona virus disease 2019 and its relationship with clinical features. Invest Radiol. 2020;55(5):257-261. 